\title{
UPPSALA NATURAL RADIOCARBON MEASUREMENTS IX
}

\author{
INGRID U. OLSSON, SHAWKY EL-GAMMAL*, \\ and YETER GÖKSU**
}

Fysiska Institutionen, Uppsala Universitet, Uppsala, Sweden

The following list covers some old measurements not included in previous lists and most of the samples measured at the Uppsala $\mathrm{C}^{14}$ laboratory since the last list (Radiocarbon, 1967, v. 9, p. 454-470); samples utilized for determining the increase of the $\mathrm{C}^{14} / \mathrm{C}^{12}$ ratio due to explosion of nuclear devices are omitted.

The technique used is mainly the same as previously described by Olsson (1958) with changes given in Radiocarbon, 1967, v. 9, p. 454. Pretreatment of samples is mainly that which has been used earlier (Radiocarbon: 1964, v. 6, p. 291; 1967, v. 9, p. 454). Since shell fragments usually are different in size, the fraction of shell samples, given in per cent as a mean value, does not give the fraction of individual shells.

The reference sample is $95 \%$ of the $\mathrm{C}^{14} / \mathrm{C}^{12}$ ratio of the NBS oxalicacid standard. Any corrections for apparent water ages are thus not included here, but are discussed in papers dealing with the samples. Corrections for deviations from the normal $\mathrm{C}^{13} / \mathrm{C}^{12}$ ratio $(-25.0 \%$ in the PDB scale) are applied for the unknown samples. Our six oxalic-acid samples have not shown any significant difference in their $\mathrm{C}^{13} / \mathrm{C}^{12}$ ratio. Our secondary standard, oxalic-acid 1, was measured by Craig (1961) and it had a $\delta \mathrm{C}^{13}$ value of $-18.97 \%$. This value agrees with the value to which the international oxalic-acid standard should be standardized. When $\delta \mathrm{C}^{13}$ is assumed, an error of $3 \%$ in the $\mathrm{C}^{13} / \mathrm{C}^{12}$ ratio is used.

The value $5570 \mathrm{yr}$ has been used for the half-life of $\mathrm{C}^{14}$, except in one case of geochemical interest (Table 1) where $5730 \mathrm{yr}$ was used as stated in the head. Since the samples in Table 1 all are rather young, only one sample will get a slightly changed activity value if the results are calculated with the half-life $5570 \mathrm{yr}$.

Results in this list are given B.P. (before 1950) or as an excess, $\Delta$, over the reference sample:

$$
\Delta=\delta \mathrm{C}^{14}-\left(2 \delta \mathrm{C}^{13}+50\right)\left(1+\frac{\delta \mathrm{C}^{14}}{1000}\right)
$$

where $\delta \mathrm{C}^{14}$ is the age-corrected $\mathrm{C}^{14}$ deviation from the reference sample in per mil in the year A.D. 1950, and $\delta \mathrm{C}^{13}$ is the deviation from PDB standard in per mil. Errors include standard deviation $(\sigma)$ of counted particles and errors in corrections due to the $\mathrm{C}^{13} / \mathrm{C}^{12}$ ratio, filling pressure, temperature, working voltage, barometric pressure etc. as described by Olsson $(1965,1966)$. When measured activity is lower than zero, $2 \sigma$ has been used for calculation of minimum age. When it is between zero and $2 \sigma$, net activity is increased by $2 \sigma$ for calculation of minimum age. Since the results are presented as physical measurements, no terms are included for the error in the half-life or previous $\mathrm{C}^{14} / \mathrm{C}^{12}$ variations.

\footnotetext{
* On leave from Univ. of Assuit, Assuit, Egypt.

** On leave from Middle East Technical University, Ankara, Turkey.
} 
These errors are included in special papers dealing with the samples and the interpretation of the results.

Several samples had to be diluted with $\mathrm{CO}_{2}$ from an old source to bring them to normal working pressure of the counters. This pressure has been about $2600 \mathrm{~mm} \mathrm{Hg}$ for samples with numbers lower than U-1000, proportional counter 1 , and about $1600 \mathrm{~mm} \mathrm{Hg}$ for samples with numbers from U-2000, proportional counter 4 .

\section{ACKNOWLEDGMENTS}

Descriptions of samples are based on information provided by those who collected and submitted them. Sincere thanks are due the contributors who helped in the preparation of the text. Special thanks are also due Dr. R. Ryhage and his co-workers for making the $\mathrm{C}^{13} / \mathrm{C}^{12}$ determinations; Prof. K. Siegbahn, who has made it possible to do this work at the institute; and Statens Naturvetenskapliga Forskningsråd, which has given the laboratory financial support. The authors are indebted to Miss Ditte Ekwall, Miss Birgitta Wallin, Mr. Martin Klasson, Miss Carina Ericsson and Mrs. Maud Söderman for their work in the laboratory and to the following students who assisted: Torsten Jonsson, Gunnar Andersson, Thyra Erikson, Maj-Lis Persson, Maj-Liz Larsson, Anders Edin, Ulla Lindahl and Rolf Jonsson. One of us (S. E.-G.) acknowledges a fellowship from Sept. 1967 to Aug. 1968 and one (Y.G.) a fellowship until the end of Feb. 1967 from Swedish International Development Authority through the International Seminar for Research and Education in Physics.

\section{SAMPLE DESCRIPTIONS}

I. GEOLOGic SAMPLES

\section{Indian Ocean series}

\section{A. Deep-sea cores}

Foraminifera tests from deep-sea core $156\left(07^{\circ} 14^{\prime} \mathrm{N}\right.$ Lat, $52^{\circ} 49^{\prime}$ E Long), Indian Ocean, depth $5107 \mathrm{~m}$. Coll. 1948 by Swedish Albatross Expedition (Pettersson); subm. by Eric Olausson, Inst. Oceanog. Univ. Gothenburg, Sweden. This core is rich in coccoliths except in a few horizons.

U-677. Core 156, 431.5 to $438.5 \mathrm{~cm},>62 \mu$, b

\section{$\mathbf{3 7 , 5 0 0 ~ B . c . ~}$}

$\delta C^{13}=-0.5 \%$

Core 156 , depth 431.5 to $438.5 \mathrm{~cm},>62 \mu$. Sample from coccolithpoor horizon. Comment: sample ultrasonically washed in boiled acidified distilled water, sieved, $\mathrm{pH}$ ca. 3, and dried before subm. to Uppsala. Activity of U-677 was slightly lower than that of U-678, but outer fraction was measured for a shorter time and statistical error was higher. As the 2 fractions had some activity within limit of errors, true age may be much higher than 40,000 yr (Olsson et al.. 1968). Innermost 60\% was used. 
U-678. Core 156, 431.5 to $438.5 \mathrm{~cm},>62 \mu$, a $>33,500$

Same sample as U-677; outermost $40 \%$ was used.

\section{Red Sea series}

Foraminifera tests from deep-sea core $162\left(11^{\circ} 57^{\prime} \mathrm{N}\right.$ Lat, $44^{\circ} 18^{\prime} \mathrm{E}$ Long), Red Sea, depth 883 m. Coll. 1948 by Swedish Albatross Expedition (Pettersson); subm. by Eric Olausson. Samples discussed by Olausson and Olsson (1969, Varve stratigraphy in a core from the Gulf of Aden: Palaeoecology, in press). For previous dates in this series, see Radiocarbon, 1967 , v. 9, p. $455-456$. Comment: difference between the 2 fractions is ascribed to contamination during long storage (Olsson et al., 1968).

$$
\text { U-2061. Core 162, } 306 \text { to } 319.5 \mathrm{~cm},>65 \mu, \text { b } \begin{array}{r}
4620 \pm 150 \\
2670 \text { B.c. }
\end{array}
$$

Core 162, depth 306 to $319.5 \mathrm{~cm},>65 \mu$. Sample from level where "warm" types (Globigerinella aequilateralis, Globigerinoides rubra and G. sacculifera) start (Todd, 1958). Comment: sample ultrasonically washed in boiled distilled acidified water, sieved, $\mathrm{pH} 3$ to 4 , and dried before subm. to Uppsala. Innermost $55 \%$ was used.

\section{0 в.C.

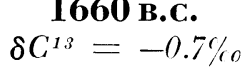

Same as U-2061; outermost $45 \%$ was used. Diluted.

\section{Pacific Ocean core series}

Foraminifera from core MSN $138 \mathrm{G}\left(00^{\circ} 15^{\prime} \mathrm{S}\right.$ Lat, $147^{\circ} 34^{\prime} \mathrm{W}$ Long). Coll. 1961 by the Monsoon Expedition. Samples were separated in different size fractions by sieving by boiled acidified distilled water $(\mathrm{pH} 4)$ and leached in HCl to give different fractions for studies of contamination such as was seen in cores from Mediterranean (Eriksson and Olsson, 1963; Olsson and Eriksson, 1965; Olsson et al., 1968). Pretreated and subm. by K. Gösta Eriksson, Inst. Geol., Univ. Gothenburg, Sweden.

$$
\begin{array}{ll}
\text { U.2114. MSN } 138 \mathrm{G}, 0 \text { to } 5 \mathrm{~cm},>62 \mu, \mathrm{b} & \begin{array}{r}
4450 \pm 160 \\
2500 \mathrm{B.c}
\end{array} \\
\delta C^{13}=+1.7 \%
\end{array}
$$

Core MSN $138 \mathrm{G}$, depth 0 to $5 \mathrm{~cm},>62 \mu$. Comment: innermost $50 \%$ was used. Diluted.

\section{U-681. MSN $138 \mathrm{G}, 0$ to $5 \mathrm{~cm}, 4$ to $45 \mu, \mathrm{b}$}

$4540 \pm 70$

2590 B.c.

Core MSN $138 \mathrm{G}$, depth 0 to $5 \mathrm{~cm}, 4$ to $45 \mu$. Comment: innermost $40^{\circ} \%$ was used.

\section{U-683. MSN $138 \mathrm{G}, 0$ to $5 \mathrm{~cm}, 4$ to $45 \mu$, a \\ $4550 \pm 100$ 2600 B.C.}

Core MSN $138 \mathrm{G}$, depth 0 to $5 \mathrm{~cm}, 4$ to $45 \mu$. Comment: $\delta C^{1.3}=+2.6,10$ $60 \%$ was used. 
4900 B.C.
$\delta C^{13}=-5.8 \%$

Core MSN $138 \mathrm{G}$, depth 5 to $10 \mathrm{~cm},>62 \mu$. Comment: innermost $5 \%$ was used. Diluted.

\section{U-2097. MSN $138 \mathrm{G}, 5$ to $10 \mathrm{~cm},>62 \mu$, a}

$4430 \pm 130$

2480 в.c.

$\delta C^{13}=-0.3 \%$

Core MSN $138 \mathrm{G}$, depth 5 to $10 \mathrm{~cm},>62 \mu$. Comment: outermost $95 \%$ was used. Diluted.

$\begin{array}{ll}\text { U.2099. MSN } 138 \mathrm{G}, 5 \text { to } 10 \mathrm{~cm}, 4 \text { to } 45 \mu, \mathrm{b} & \begin{array}{r}\mathbf{5 4 8 0} \pm \mathbf{3 5 3 0} \text { в.C. } \\ \delta C^{13}=-2.1 \%\end{array}\end{array}$

Core MSN $138 \mathrm{G}$, depth 5 to $10 \mathrm{~cm}, 4$ to $45 \mu$. Comment: innermost $30 \%$ was used, but fractionation might have failed.

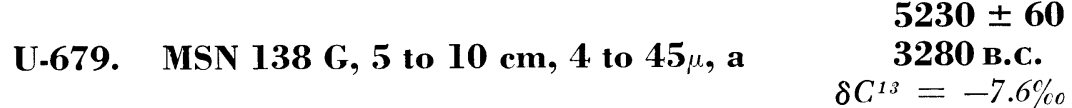

Core MSN $138 \mathrm{G}$, depth 5 to $10 \mathrm{~cm}, 4$ to $45 \mu$. Comment: outermost $70 \%$ was used.

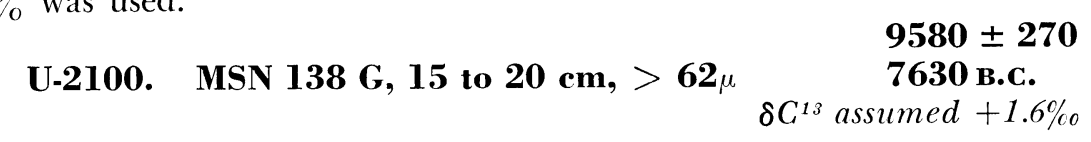

Core MSN $138 \mathrm{G}$, depth 15 to $20 \mathrm{~cm},>62 \mu$. Comment: whole sample was used. Diluted.

$\begin{array}{ll}\text { U-2098. MSN } 138 \mathrm{G}, 15 \text { to } 20 \mathrm{~cm}, 4 \text { to } 45 \mu, \text { b } & 9730 \pm 310 \\ 7780 \text { в.c. }\end{array}$

$\delta C^{13}=-3.4 \%$

Core MSN $138 \mathrm{G}$, depth 15 to $20 \mathrm{~cm}, 4$ to $45 \mu$. Comment: innermost $25 \%$ was used. Diluted.

\section{U-680. MSN $138 \mathrm{G}, 15$ to $20 \mathrm{~cm}, 4$ to $45_{\mu}$, a}

$9750 \pm 100$ 7800 B.C.

$\delta C^{13}=-3.5 \%$

Core MSN $138 \mathrm{G}$, depth 15 to $20 \mathrm{~cm}, 4$ to $45 \mu$. Comment: outermost $75 \%$ was used.

General Comment: only one sample, U-2097, shows significant deviation from other fractions of same level in core.

\section{Sogho-nor series}

B. Asia

Plant remains from Lake Sogho-nor $\left(42^{\circ} \mathrm{N} \mathrm{Lat}, 101^{\circ} \mathrm{E}\right.$ Long). Samples coll. by Hörner; subm. by E. Norin, Inst. Geol., Univ. Uppsala, Sweden. Samples from strand-line now $8.6 \mathrm{~m}$ above present level. Comment: oasis of Etsina was flourishing ca. $600 \mathrm{yr}$ ago; results show that water 
level then was ca. $8 \mathrm{~m}$ higher than now and lake much larger. Described by Norin (1966).

\section{U-283. Sogho-nor 270530}

A.v. 1450

$\mathbf{5 0 0} \pm \mathbf{3 0 0}$

$\delta C^{13}=-16.0 \%$

Aquatic plants, mainly, coll. in highest ancient beach ridge, $7.5 \mathrm{~m}$ above water level. Sample SW of lake. Coll. 1930. Comment: diluted.

\section{U-284. Sogho-nor 150333:1}

\section{A.D. 1280}

$$
670 \pm 110
$$

$\delta C^{13}=-14.7 \%$

Aquatic plants, mainly, coll. from ancient beach, probably $8 \mathrm{~m}$ above level of lake. Sample SE of lake. Coll. 1933. Comment: diluted.

\section{U-273. Sogho-nor $150333: 2$}

$$
\begin{aligned}
& 660 \pm 120 \\
& \text { A.D. } 1290 \\
& \delta C^{13}=-15.7 \%
\end{aligned}
$$

Same sample and pretreatment as U-284, but new combustion. Comment: diluted.

\section{U-2036. Elmau}

\section{Central Europe}

$$
\begin{aligned}
& >\mathbf{3 4 , 6 0 0} \\
\delta C^{13} & =-25.6 \% \circ
\end{aligned}
$$

Tree root from adit Elmau (ca. $47^{\circ} 25^{\prime} \mathrm{N}$ Lat, $13^{\circ} 05^{\prime} \mathrm{E}$ Long), Mühlbach/Hochkönig, Salzburg, Austria. Trees were growing on slope of former valley, now filled by gravel and breccia ca. $80 \mathrm{~cm}$ thick. Coll. by M. Maczek, Kupferbergbau Mitterberg, Austria.

\section{River Ula basin series}

\section{Lithuanian S.S.R.}

Wood from Rudnja (54 $\mathrm{N}$ Lat, $25^{\circ} \mathrm{E}$ Long), Ula R. basin, $70 \mathrm{~km}$ $\mathrm{S}$ Vilnius, Lithuania. Material from this area has been dated previously, Mo-302, Zervinos (moss): 16,260 \pm 640 ; Mo-339, Rudnja (peat): 12,715 \pm 315; Mo-341, Pamärkis (peat): 11,500 \pm 430 ; Mo-340, Pamärkis (wood): $12,260 \pm 160 ;$ Vs-4, Zervinos (peat): 18,350 \pm 950; Vs-5, Manciagire (peat): 17,340 \pm 840; TA-124, Zervinos (wood): 11,930 \pm 110 ; TA-125, Zervinos (moss): 12,160 \pm 120 ; TA-191, Zervinos (moss): 12,650 \pm 130 ; TA-190, Rudnja (wood): 11,530 \pm 120 (Simm, pers. commun.). Samples are deposits submerged under fluvioglacial and eolian sand; they are suggested either to be interstadial or of late-glacial origin. Wood of same tree trunk used for TA-190, subm. as interlaboratory check by H. Simm, Zool. Bot. Inst., Eesti NSV Teaduste Akad., Tartu, Estonia S.S.R.

\section{U.675. Rudnja, 2}

Wood from Rudnja, 0.39 to $0.93 \mathrm{~m}$ below surface. Sample coll. in aleuritic sand with peat and wood. 


\section{U-2107. Rudnja, 1}

Wood from same sample as was used for $\begin{aligned} & \delta C^{13} \text { assumed }-675 \text {, but new pre- } \\ & \text { W }\end{aligned}$ treatment.

\section{Shoreline series}

$$
\text { E. Iceland }
$$

Shell and wood from Iceland to determine chronology of Late- and Post-glacial shorelines. Coll. 1966 and subm. by Thorleifur Einarsson, Univ. Research Inst., Reykjavik, Iceland.

U.641. Melar, 3-67, b

$$
\begin{gathered}
12,290 \pm 160 \\
10,340 \text { в.C. } \\
\delta C^{13}=+0.2 \%
\end{gathered}
$$

One valve (Pecten islandicus) from Melar $\left(64^{\circ} 25^{\prime} \mathrm{N}\right.$ Long, $22^{\circ} 01^{\prime}$ W Long), Melasveit, Iceland, alt $5 \mathrm{~m}$ (Bárdarson, 1923). Sample from glaciomarine silt, which seems to interfinger with an end moraine of Alftanes Readvance, which probably is of Older Dryas age. Comment: similar sample, U-2019, dated: 11,620 \pm 240 B.P. (Radiocarbon, 1967, v. 9, p. 458 , where preliminary result was given). Innermost $60 \%$ was used.

\section{U.2054. Melar, 3-67, a}

$12,610 \pm 200$

\section{0,660 в.C.}

$\delta C^{13}=+0.2 \%$

Shell layer surrounding part used for U-641. Comment: outermost $40_{\%}^{\circ}$ was used.

\section{U-640. Hólahólar, 5-67}

$6940 \pm 110$ 4990 B.C.

Juniperus communis from Hólahólar $\left(\begin{array}{lll}65^{\circ} & 20^{\prime} \mathrm{N} & \delta C^{13}=-27.0 \% \\ \hline & \text { Lat, } 18^{\circ} 10^{\prime} \mathrm{W}\end{array}\right.$ Long), Eyjafjördur, Iceland, alt $60 \mathrm{~m}$. Sample from rockslide, cut through by the Eyjafjardlará R. Sample supposed to be older than Hekla ash laver $\mathrm{H}_{3}$. Pollen Zone A or B from N Iceland (Einarsson, 1961).

\section{U-2088. Baejará, 2-67}

$3140 \pm 140$

1190 B.C.

$\delta C^{13}=0.0 \%$

Astarte borealis (L) from Baejará (65 $15^{\circ} \mathrm{N}$ Lat, $21^{\circ} 11^{\prime} \mathrm{W}$ Long), Hrútafjördur, Iceland, alt $3 \mathrm{~m}$. Sample from top of gravel bar, $2.5 \mathrm{~m}$ higher than present one, $35 \mathrm{~m}$ inland and covered by ash layer $\mathrm{H}_{4}$. assumed 4000 B.P. (Bárdarson, 1910; Thorarinsson, 1955). Comment: innermost $90 \%$ was used. Diluted.

\section{U-2082. Tjarnargata $4,6-67$}

$1140 \pm 70$

\section{A.D. 810}

$\delta C^{13}=-26.8^{\%} \%$

Wood shavings from Tjarnargata 4 , Reykjavik $\left(64^{\circ} 09^{\prime} \mathrm{N}\right.$ Lat, $21^{\circ}$ $57^{\prime} \mathrm{W}$ Long), Iceland. Sample in peat with wood fragments and bones (Sus scrofa, domesticus, Alca impermis, and Trichechis rosmarus). Coll. 
1944 by Finnur Gudmundsson, Mus. Nat. Hist., Reykjavik, Iceland and subm. by Th. Einarsson. Comment: another sample from old cultural layer nearby was dated previously: K-940, $1340 \pm 100$ (Radiocarbon, 1966, v. 8, p. 232). Diluted.

\section{U-632. Ellidaár 650609}

\section{$4630 \pm 90$ 2680 B.c.}

Charred pieces of birch from Ellidaár $\left(64^{\circ} 08^{\prime} \mathrm{N}\right.$ Iat, $21^{\circ} 50^{\prime}$ W Long), Reykjavik, Iceland. Sample underlying lava, Leitahraun, and overlying Paruocaricetum peat. Coll. 1965 and subm. by Jón Jónsson, State Electricity Authority, Reykjavik, Iceland. Lava described by Hospers (1953). Comment: peat nearby was dated previously: C-749, 5300 \pm 340 years (Libby, 1955).

\section{U-2051. Dýrholaós}

$$
\begin{aligned}
& \quad 1240 \pm 100 \\
& \text { A.D. } 710 \\
& \delta C^{13}=-23.7 \%
\end{aligned}
$$

Driftwood from Dýrholaós (63 $20^{\prime} \mathrm{N}$ Lat, $19^{\circ} 05^{\prime} \mathrm{W}$ Long), Skaftafellssýsla, Iceland. Sample from a log, $3 \mathrm{~m}$ long, diam. 25 to $30 \mathrm{~cm}$, imbedded in peat, now at a lagoon. Due to scarcity of timber on Iceland, logs at this locality presumed to have been imbedded before colonization. Thickness of peat also implies that sea level of the time was several $\mathrm{m}$ lower than now. Driftwood deposited at extreme highwater. Coll. and subm. by Jón Jónsson.

\section{F. Spitsbergen, Björnöya, and Shetland}

\section{Vestspitsbergen series}

Driftwood, shells, and whale bones from Vestspitsbergen coll. for determination of land uplift. Alt. given above mean sea level. Previous determinations in this series are given in Radiocarbon: 1960, v. 2, p. $115-116$; 1961, v. 3, p. 82-83; 1964, v. 6, p. 296-298; 1965, v. 7, p. 317-318; 1967 , v. 9, p. 456-457. Quaternary geology and land uplift is discussed by Birkenmajer (1960), Feyling-Hanssen (1955), Feyling-Hanssen and Olsson (1959-1960). Samples from Hornsund area coll. 1966 and subm. by Krzysztof Birkenmajer, Polish Acad. Sci., Krakow, Poland and those from Isfjorden area coll. 1960 by D. H. Maling. Comment: bone treatment with EDTA is described in Radiocarbon, 1967, v. 9, p. 456.

\section{U.619. Rettkvalbogen E, 1 st, Q4a}

$1080 \pm 70$ A.D. 870

$\delta C^{13}=-23.7 \%$

Driftwood from Rettkvalbogen E $\left(77^{\circ} 00^{\prime} \mathrm{N}\right.$ Lat, $15^{\circ} 32^{\prime} 30^{\prime \prime} \mathrm{E}$ Long), Hornsund, Spitsbergen, alt. $5.5 \mathrm{~m}$. Sample from $\log$, $10 \mathrm{~m}$ long, $40 \mathrm{~cm}$ diam., partly buried in moss, 1st terrace.

\section{U-2048. Rettkvalbogen E, 1 st, Q5a, R}

$1120 \pm 80$ A.D. 830 $\delta C^{13}=-19.5 \%$

Balaena mysticetus L. from Rettkvalbogen E $\left(77^{\circ} 00^{\prime} \mathrm{N}\right.$ Lat, $15^{\circ}$ 
$32^{\prime} 30^{\prime \prime}$ E Long), Hornsund, Spitsbergen, alt $5.5 \mathrm{~m}$. Sample from almost complete upper left jaw, $5.6 \mathrm{~m}$ long, $0.45 \mathrm{~m}$ wide in its rear edge, partly buried in moss, 1st terrace. Comment: sample treated with acetone before EDTA extraction.

\section{U-620. Rettkvalbogen E, 1 st, Q5a, A}

Acetone extract from sample used for U-2048.

U-2079. Fuglebekken, 2a, Q6a, b
$770 \pm 70$

$$
\text { A.D. } 1180
$$$$
\delta C^{13}=-24.3 \%
$$

$$
9210 \pm 180
$$

7260 B.C.

$\delta C^{13}$ assumed $-2.1 \%$

Fragments (Mya truncata and Astarte borealis) from Fuglebekken $\left(77^{\circ} 00^{\prime} 30^{\prime \prime} \mathrm{N}\right.$ Lat, $15^{\circ} 34^{\prime} \mathrm{E}$ Long), Hornsund, Spitsbergen, alt $5 \mathrm{~m}$. Sample in sandy gravel, Horizon "a" of $2 \mathrm{a}_{1}$ Mya Terrace. Comment: innermost $33 \%$ was used.

U.2069. Fuglebekken, 2a, Q6a, a

$9150 \pm 160$ 7200 B.C.

$$
\delta C^{13}=-2.1 \%
$$

Shell layer surrounding part used for U-2079. Comment: layer corresponds to $37 \%$ of shells; $30 \%$ we removed by washing.

U-682. Marmorneset, 2a, Q1a, b

$7410 \pm 90$ 5460 в.c.

$$
\delta C^{13}=+0.3 \%
$$

Shell fragments, mostly Balanus, from $\mathrm{W}$ part of Marmorneset $\left(77^{\circ}\right.$ $00^{\prime} \mathrm{N}$ Lat, $15^{\circ} 29^{\prime} \mathrm{E}$ Long), Hornsund, Spitsbergen, alt $8 \mathrm{~m}$. Sample in shingle and subrounded gravel, with sandy matrix, Horizon "f" of $2 \mathrm{a}_{\text {. }}$ Saxicava- Mya Terrace. Comment: innermost $55 \%$ was used.

\section{U.2065. Marmorneset, 2a, Q1a, a}

$7620 \pm 130$

5670 B.c.

$\delta C^{13}=+0.2 \%$

Shell layer surrounding part used for U-648. Comment: layer corresponds to $20 \%$ of shells; $25 \%$ was removed by washing.

\section{U-665. Isbjørnhamna, 2a, Q3a, b}

$9620 \pm 110$

7670 B.C.

$\delta C^{13}=+0.2 \%$

Fragments (Mya truncata and Saxicava arctica) from Isbj $\phi$ rnhamna $\left(77^{\circ} 00^{\prime} \mathrm{N}\right.$ Lat, $15^{\circ} 33^{\prime} 30^{\prime \prime}$ E Long), Wilczekodden, Hornsund, Spitsbergen, alt. $7.5 \mathrm{~m}$. Sample overlay sand and gravel but underlay sand, Horizon "d" of $2 \mathrm{a}_{2}$ Terrace, Saxicava-Mya Terrace. Comment: innermost $50 \%$ was used.

\section{U-666. Isbjørnhamna, 2aa, Q3a, a}

Shell layer surrounding part used for U-665. Comment: layer corresponds to $30 \%$ of shells; $20 \%$ was removed by washing. 
U.703. Isbjørnhamna, 2a 2 Q2a, RI

$9380 \pm 140$

7430 в.c.

$\delta C^{13}=-17.1 \%$

Whale bone (probably Balaena mysticetus L.) from Isbjørnhamna ( $77^{\circ} 00^{\prime} \mathrm{N}$ Lat, $15^{\circ} 33^{\prime} 30^{\prime \prime}$ E Long), $82 \mathrm{~m}$ SW Polish Sta., Hornsund, Spitsbergen, alt $8 \mathrm{~m}$. Sample from lower jaw, $1.9 \mathrm{~m}$ long, $17 \mathrm{~cm}$ diam, $2 / 3$ buried in tundra: mosses, lichens, Saxifraga, Salix polaris, and grass, $2 \mathrm{a}_{2}$ Terrace. Comment: EDTA-treated after acetone extraction.

U-2130. Isbjørnhamna, 2a $\mathbf{a}_{2}, \mathbf{Q 2 a}, \mathbf{R 2}$

$9840 \pm 230$

7890 в.C.

$\delta C^{13}=-18.2 \%$

Gas from another combustion but from same pretreatment as for sample U-703. Diluted.

\section{U-2131. Isbjørnhamna, 2a, Q2a, W}

$8610 \pm 170$

660 в.c.

$\delta C^{13}=-19.4^{\%} \%$

Wrong fraction from EDTA-treatment of sample U-703 and U-2130.

\section{U-2077. Ekholmvika 6020 b, R}

$9650 \pm 130$

7700 B.c.

$\delta C^{13}=-19.4 \%$

Whale rib from Ekholmvika ( $78^{\circ} 35^{\prime} \mathrm{N}$ Lat, $16^{\circ} 38^{\prime} \mathrm{E}$ Long), Billefjorden, Spitsbergen, alt ca. $50 \mathrm{~m}$. Sample protruding from surface and buried $1 \mathrm{~m}$ in coarse gravel. Location mapped by Balchin (1941). Comment: sample treated with acetone before EDTA-treatment.

\section{U-2066. Ekholmvika $6020 \mathrm{~b}$, inorganic $\quad \Delta=1270 \pm \mathbf{3 6} \%$} $\delta C^{13}$ assumed $-5.2 \%$

Same rib as U-2077 but inorganic fraction, $\mathrm{CO}_{2}$ liberated at $\mathrm{HCl}$ treatment under vacuum, assumed to give false result. Comment: diluted. Sample contaminated by $\mathrm{CO}_{22}$ of air in lab.

\section{U-2076. Ekholmvika 6020 b, HCl-NaOH}

$$
\begin{gathered}
3500 \\
\mathbf{1 5 5 0 ~ B . C .} \\
\delta C^{13}=-24.8 \% \text {.410 }
\end{gathered}
$$

Insoluble remains from $\mathrm{HCl}$ treatment giving U-2066. Remains treated with $0.5 \% \mathrm{NaOH}$, washed, made acid and dried before combustion. Comment: diluted.

\section{U-2121. Ekholmvika 6020 b, HCI}

$5990 \pm 380$

4040 в.c.

$\delta C^{13}$ assumed $-19.0 \%$

Insoluble remains of same rib as $\mathrm{U}-2066,2076$, and 2077, after treatment of crushed bones with $1-\mathrm{N} \mathrm{HCl}$ until $\mathrm{pHl}$ and washing with distilled water before drying. Comment: diluted. 


\section{U-2106. Ekholmvika $6021 \mathrm{~b}, \mathbf{R}$}

Whale rib from Ekholmvika ( $78^{\circ} 35^{\prime} \mathrm{N}$ Lat, $16^{\circ} 38^{\prime} \mathrm{E}$ Long), Billefjorden, Spitsbergen, alt ca. $50 \mathrm{~m}$. Sample protruding ca. $0.75 \mathrm{~m}$ from surface and easily broken.

\section{Nordaustlandet series}

Wood and shells for determination of land uplift. Previous samples in this series are given by Olsson and Blake (1961-1962) and in Radiocarbon, 1959, v. 1, p. 90-91; 1960, v. 2, p. 116-121; 1964, v. 6, p. 298-300. Coll. and subm. by W. Blake, Jr., Geol. Surv. of Canada, Ottawa, Ontario, Canada, except for K. Sparre-Sveanor samples.

\section{U-618. Kvalrosshalvфya 174-66}

$6700 \pm 70$

4750 в.C.

$\delta C^{13}=-23.7 \%$

Picea or Larix from Kvalrosshalv $\phi y a\left(79^{\circ} 59^{\prime} \mathrm{N}\right.$ Lat, $18^{\circ} 35^{\prime} \mathrm{E}$ Long), Nordaustlandet, Spitsbergen, alt $9.8 \mathrm{~m}$. Sample partly buried in beach shingle with pumice. Subm. to check suggestion (Olsson and Blake, 19611962) that U-33 and U-34 dated 9.8 and $2.0 \mathrm{~m}$ levels, respectively, instead of the contrary. U-618 is from same $\log$ as U-33 $(6910 \pm 110)$. Coll. 1966.

\section{U.2058. Lindhagenbukta 71-66, b}

Macoma calcarea from Lindhagenbukta $\left(80^{\circ} 17^{\prime} \mathrm{N}\right.$ Lat, $20^{\circ} 42^{\prime} \mathrm{E}$ Long), Nordaustlandet, Spitsbergen, alt 0 to $2 \mathrm{~m}$. Sample from assemblage of well-preserved shells, still hinged and with periostracum nearly intact. Sample supposed to give apparent age of water; shells assumed to be only a few yr old as they had not been crushed by rafted ice. Coll. 1966. Comment: apparent age of water $420 \mathrm{yr}, \Delta=-50 \pm 10 \%$, if sample assumed from 1960. Innermost $40 \%$ was used.

\section{U-2059. Lindhagenbukta 71-66, a}

$380 \pm 90$

A.D. 1570

$\delta C^{13}=-1.6 \%$

Shell layer surrounding U-2058. Comment: apparent age $390 \mathrm{yr}$; $\Delta=-47 \pm 10 \%$. Outermost $60 \%$ of shells was used.

\section{U.646. Lady Franklinfjorden 163-66, b}

$$
22,600 \pm 500
$$

$$
\begin{aligned}
& 20,600 \text { в.c. } \\
& \delta C^{13}=+5.3 \%
\end{aligned}
$$

Fragments (Hiatella arctica, Mya truncata, and possibly Macoma and Chlamys) from Kapp Lady $\left(80^{\circ} 12^{\prime} \mathrm{N}\right.$ Lat, $18^{\circ} 42^{\prime} \mathrm{E}$ Long), Lady Franklinfjorden, Nordaustlandet, Spitsbergen, alt ca. $2 \mathrm{~m}$; depth ca. 0.5 $\mathrm{m}$, from till overlain by beach shingle with lenses of organic debris between till and gravel. Surface of shells pitted but sample dated to check 
U-263 (Radiocarbon, 1964, v. 6, p. 299) from same place. Both dates thought by senior author to give unrealistically low ages. Coll. 1966. Comment: innermost $60 \%$ was used. \\ U-2060. Lady Franklinfjorden, 163-66, a $-\mathbf{8 0 0}$ \\ $\delta C^{13}=+5.9 \%$}

Shell layer surrounding U-646. Comment: layer corresponds to $30 \%$ of sample. $10 \%$ was removed by washing.

\section{U-2095. K. Sparre-Sveanor, c}

$11,180 \pm 190$ 9230 в.c.

Mya truncata from K. Sparre-Sveanor $\left(79^{\circ} 56^{\prime} \mathrm{N}\right.$ Lat, $18^{\circ} 15^{\prime} \mathrm{E}$ Long), Nordaustlandet, Spitsbergen, alt $82 \mathrm{~m}$. Shells partly buried in beach material. Coll. 1966 by M. Grosswald; subm. by G. Hoppe. Comment: shells from same collection previously dated, St-2374: 10,670 \pm 145. Innermost $35 \%$ was used.

U-660. K. Sparre-Sveanor, b

$11,150 \pm 110$ 9200 B.C.

$\delta C^{1,3}$ assumed $+0.3 \%$ of shells.

Shell layer surrounding U-2095. Comment: layer corresponds to $40 \%$

U-661. K. Sparre-Sveanor, a

$10,370 \pm 220$

8420 B.C.

$\delta C^{13}$ assumed $+0.3 \%$

Shell layer surrounding U-2095 and U-660. Comment: layer correspondls to $25 \%$ of shells.

\section{Lake sediment series; Nordaustlandet, Bjørnфya, and Shetland}

Silty mud from Skinkevatnet ( $74^{\circ} 30^{\prime} \mathrm{N}$ Lat, $19^{\circ} 00^{\prime} \mathrm{E}$ Long), Bj $\phi \mathrm{r}^{-}$ nóya, alt $19.3 \mathrm{~m}$; clay-gyttja from Rijpljorclen $\left(80^{\circ} 18^{\prime} \mathrm{N}\right.$ Lat, $22^{\circ} 40^{\prime}$ E Long), Nordaustlandlet, Spitsbergen, alt $30 \mathrm{~m}$; gyttja from tidal lake Lower Loch of Brouster $\left(60^{\circ} 15^{\prime} \mathrm{N}\right.$ Lat, $01^{\circ} 36^{\prime} \mathrm{W}$ Long), Shetland; graphite from chemical store. Geologic samples supposedly contain old carbon and different separation methods are being tried. Bjornфya samples coll. 1965, subm. and described by Hannu Hyvärinen (1968); Nordaustlandet sample coll. 1965 and subm. by Hyvärinen, Dept. Geol. and Palaeontol. Univ. Helsinki, Finland; Shetland sample coll 1964, subm. and described by Gunnar Hoppe (1965), Dept. Physical Geog. Univ. Stockholm, Sweden; chemical treatment is discussed by Olsson in a preliminary report (1968). Comment: all samples diluted-in many cases more than can be recommended, so that errors are so large that result is of little geologic interest. 
U-2031. Skinkevatnet, 92.5 to 87.5, H

$11,200 \pm 500$

9200 B.C.

$\delta C^{13}=-25.4 \%$ o

Humus products extracted from sample, 92.5 to $87.5 \mathrm{~cm}$ depth.

$$
8300 \begin{aligned}
& +1400 \\
& -1200
\end{aligned}
$$

U-2042. Skinkevatnet, 92.5 to 87.5, $\mathrm{HNO}_{3}+\mathrm{H}_{2} \mathrm{O}_{2} \quad 6300$ в.c.

$$
\delta C^{13}=-25.3 \%
$$

Gas obtained by wet combustion of sample, with $\mathrm{HNO}_{3}+\mathrm{H}_{2} \mathrm{O}_{2}$, after alkali extraction that gave U-2031.

\section{U-2064. Skinkevatnet, 92.5 to 87.5 , INS}

$$
\begin{gathered}
8900 \\
\text { 7000 B.c. }
\end{gathered}
$$

$\delta C^{13}$ assumed $-25.4 \%$

Insoluble remains of sample, after wet combustion that gave U-2042.

U-2050. Skinkevatnet, 42.5 to $37.5, \mathrm{H}$

$$
\begin{gathered}
\mathbf{4 6 0 0}{ }^{+1200} \\
-\mathbf{1 0 0 0} \\
\mathbf{2 7 0 0 \text { B.C. }} \\
\delta C^{13}=-28.2 \% 0
\end{gathered}
$$

Humus products extracted from sample, 42.5 to $37.5 \mathrm{~cm}$ depth.

\section{U-2125. Skinkevatnet, 42.5 to $37.5, \mathrm{KMnO}_{4} \quad 5300$ B.C.}

$$
7300+1100
$$

Gas obtained by wet combustion of sample, with $\mathrm{H}_{2} \mathrm{SO}_{4}+\mathrm{KMnO}_{4}$, after extraction that gave $\mathrm{U}-2050$.

U-2128. Skinkevatnet, 42.5 to 37.5 , INS

$9460 \pm 350$

7510 B.C.

$\delta C^{13}=-21.7 \%$

Insoluble remains of sample, after wet combustion that gave U-2125.

U-2049. Skinkevatnet, 22.5 to $17.5, \mathrm{H}$

$$
\begin{aligned}
& 6000 \\
& +3100 \\
& -2200 \\
& \delta C^{13}=-23.9 \%
\end{aligned}
$$

Humus products extracted from sample, 22.5 to $17.5 \mathrm{~cm}$ depth.

U-2124. Skinkevatnet, 22.5 to $17.5, \mathrm{KMnO}_{4}$

Gas obtained by wet combustion of sample, with $\mathrm{H}_{2} \mathrm{SO}_{4}+\mathrm{KMnO}_{4}$, after extraction that gave U-2049. 


\section{U-2127. Skinkevatnet, 22.5 to 17.5 , INS}

$6870 \pm 470$

4920 в.c.

$\delta C^{13}=-21.7 \%$

Insoluble remains of sample, after wet combustion that gave U-2124.

\section{U-2110. Rijpfjorden $3 / 23,60$ to $55, \mathrm{H}$}

$$
10,150 \pm 450
$$

Humus products extracted from sample, 60.5 to $54.5 \mathrm{~cm}$ depth. Comment: another sample immediately underlying that used for U-2110 was dated previously, St-2532: 10,885 \pm 250 .$$
9640
$$

U-2126. Rijpfjorden $3 / 23,60$ to $55, \mathrm{KMnO}_{4}$

Gas obtained by wet combustion of sample, with $\mathrm{H}_{2} \mathrm{SO}_{4}+\mathrm{KMnO}_{4}$, after extraction that gave U-2110.
}

\section{U-2129. Rijpfjorden $3 / 23,60$ to 55 , INS}

$$
\delta C^{13}=-25.3 \%
$$

Insoluble remains of sample, after wet combustion that gave U-2126.

U-616. Lower Loch of Brouster, Core 20,

$$
\begin{array}{r}
6300 \\
4350 \text { B.c. } \\
\text { 4350 } \\
\delta C^{13}=-20.0 \%
\end{array}
$$
343 to 338, H, I

Humus extracted from gyttja, 343 to $338 \mathrm{~cm}$ below top of sediment. Comment: another sample from same core, $5 \mathrm{~cm}$ lower, was dated previously (U-576 and U-2007, mean $7650 \pm 450$; Radiocarbon, 1967, v. 9, p. 460$)$.

U-2041. Lower Loch of Brouster, Core 20, 343 to 338, $\mathrm{HNO}_{3}+\mathrm{H}_{2} \mathrm{O}_{2}$

Gas obtained by wet combustion of sample, with $\mathrm{HNO}_{3}+\mathrm{H}_{2} \mathrm{O}_{2}$, after extraction that gave U-616.

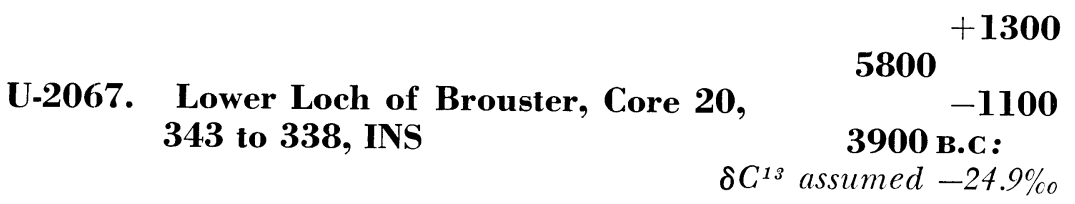

Insoluble remains of sample, after wet combustion that gave U-204l. 
Graphite from dealer in chemicals, Kebo. Comment: gas obtained by wet combustion with $\mathrm{H}_{2} \mathrm{SO}_{4}+\mathrm{KMnO}_{4}$.

\section{G. Norway}

\section{U.2121. Sandnes 1966, No. 11, R}

$$
\delta C^{13}=-13.9 \%
$$

Balaena mysticetus from Sandnes (58 $50^{\prime} \mathrm{N}$ Lat, $05^{\circ} 42^{\prime} \mathrm{E}$ Long), Rogaland, Norway, $10 \mathrm{~m}$ deep in glaciomarine clay with Portlandia arctica etc., at ca. $17 \mathrm{~m}$ depth. Clay overlain by sand and Würm till. Coll. 1966 and subm. by Björn Andersen, Inst. Geol. Univ. Oslo, Blindern, Norway. Comment: sample EDTA-treated. Another piece of same bone dated previously after normal $\mathrm{HCl}$ treatment: T-641, 28,900 \pm 800( Andersen, written commun.). Diluted.

\section{Skjomen series}

Gyttja from 2 tarns at defile at Somarskardet $\left(68^{\circ} 17^{\prime} \mathrm{N}\right.$ Lat, $16^{\circ}$ 73' E Long), near fault scarp, SW Narvik, Nordland, Norway, alt ca. $+600 \mathrm{~m}$. Samples coll. to correlate lateral moraines in area with position of Tromsö-Lyngen substage ice margin. Geology discussed by Dahl (1967, 1968). Coll. 1966 and subm. by Ragnar Dahl, Dept. Physical Geog., Univ. Uppsala, Sweden. Comment: differences between humus-products and insoluble remains are, for 2 of the samples, rather large although samples were so small that they had to be diluted; errors, thus, are large. Dilution for U-605 and U-2028 was larger than normally allowed. Taking previous variations of $\mathrm{C}^{14} / \mathrm{C}^{12}$ into account, large differences will decrease, however.

\section{U-605. Skjomen I:1, 0 to 5 , INS}

Gyttja from lower $5 \mathrm{~cm}$ of $80 \mathrm{~cm}$ thick organic sediments of $1 \mathrm{tarn}$, $250 \mathrm{~m} \mathrm{~N}$ of threshold, $\mathrm{E}$ of and near moraine ridge.

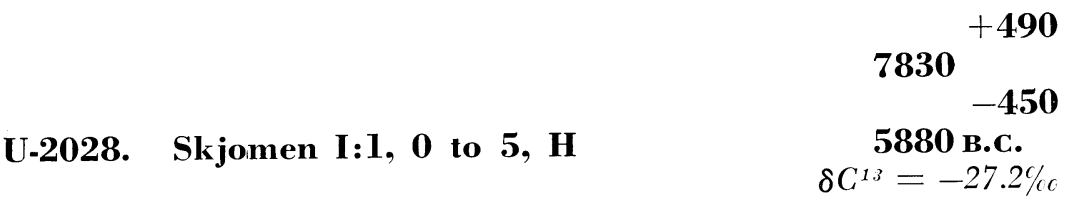

Humus products extracted from sample used for U-605.

\section{U-2037. Skjomen I:2, 5 to 10, INS}

$3650 \pm 110$

1700 B.c.

$\delta C^{13}=-22.9 \%$

Gyttja 5 to $10 \mathrm{~cm}$ above sand and silt bottom of tarn from which U-605 + U-2028 was coll. 
Humus products extracted from sample used for U-2037.

\section{U-2038. Skjomen I:2, 5 to $10, \mathrm{H}$}

$3210 \pm 100$

\section{U-2039. Skjomen I:3, 0 to 5 , INS}

1260 в.C.

$\delta C^{13}=-25.8 \%$

Gyttja from lower $5 \mathrm{~cm}$ of $30 \mathrm{~cm}$ thick organic sediments of tarn, $\mathrm{W}$ of moraine ridge near tarn where Sample U-605 was coll.

\section{U-2040. Skjomen I:3, 0 to $5, \mathrm{H}$}

$3390 \pm 230$

1440 B.c.

Humus products extracted from sample used for U-2039.

$$
\delta C^{13}=-27.8 \%
$$

\section{H. Sweden}

\section{U-2071. Levide, TF 67}

$8920 \pm 190$

6970 B.C.

$\delta C^{13}=-25.0^{\prime} / \%$

Wood from trunk (Pinus) from Levide $\left(57^{\circ} 15^{\prime} \mathrm{N}\right.$ Lat, $18^{\circ} 15^{\prime} \mathrm{E}$ Long), Gotland, Sweden. Sample from sand and gravel, $2 \mathrm{~m}$ below surface, with Ancylus shells and several other trunks. Comment: 12 outer tree rings of log, containing 50 tree rings, were used. Coll. 1967 and subm. by Trygve Fahlstedt, Dept. Agr. Hydrotechnics, High School of Agric. Ultuna, Uppsala, Sweden. Comment: another sample from Levide was dated previously, U-428: $9830 \pm 140$ (Radiocarbon, 1964, v. 6, p. 301).

\section{Shell bank series}

Shells from Lindalsskogen, Väjern ( $58^{\circ} 22^{\prime} \mathrm{N}$ Lat, $06^{\circ} 47^{\prime} \mathrm{E}$ Long), Bohuslän, Sweden, alt $25 \mathrm{~m}$ and at one unspecified place in Bohuslän, Sweden; shells from Väjern coll. 1966 in shell banks subm. by K. Gösta Eriksson, Inst. Geol. Univ. Gothenburg, Sweden. Samples discussed by Eriksson and Olsson (1967).

\section{U.2029. Väjern 3282 B}

$$
\begin{gathered}
10,160 \pm 330 \\
8210 \text { B.C. } \\
\delta C^{13} \text { assumed }-1.0 \% \text {. }
\end{gathered}
$$

Shells (Balanus balanus) coll. 0.2 to $1 \mathrm{~m}$ below erosion layer at alt. ca. $24 \mathrm{~m}$. Comment: innermost $30 \%$ was used. Diluted.

\section{U-2030. Väjern 3282 H}

$$
\begin{gathered}
10,230 \pm 240 \\
\mathbf{8 2 8 0} \text { в.c. } \\
\delta C^{13}=+1.4 \%
\end{gathered}
$$

Shells (Balanus hameri) 0.2 to $1 \mathrm{~m}$ below erosion layer. Comment: innermost $55 \%$ was used. Diluted. 


\section{U-607. Bohuslän}

$$
\begin{aligned}
& \mathbf{5 3 0} \pm \mathbf{8 0} \\
& \text { A.D. } 1420 \\
& \delta C^{1.3}=-1.7 \% c
\end{aligned}
$$

Valve (Patella vulgata) from Bohuslän coll. ca. 1935 by Ingrid U. Olsson. Apparent age: 510 yr. $\Delta=-62 \pm 9 \%$. Comment: innermost $95 \%$ was used. Diluted.

\section{Degerfors series}

Gyttja from Svea Älv (Svea R.) area at Degerfors. Coll. 1967 and subm. by Curt Fredén, Inst. Quaternary Geol., Univ. Uppsala, Sweden. Coll. with Livingstone borer. Pollen analyses by Fredén. Survey of previous discussions on Svea R. given by Fredén (1967). Samples described by Olsson et al. (in press).

\section{U-2080. Håkanbol 7, INS}

$8060 \pm 220$

6110 B.C. $14^{\circ} 2^{\prime}$ E surface in kettle hole filled by peat. Zone $\mathrm{V}$ (Jessen). Before birch culmination. Assumed older than Svea R. Comment: diluted.

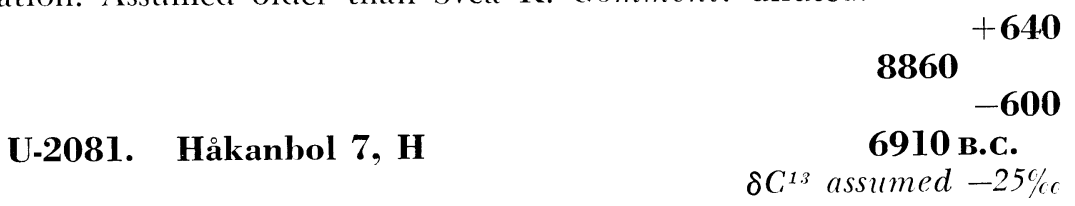
diluted.

Humus products extracted from sample used for U-2080. Comment:

\section{U.2113. Håkanbol 18, INS}

$8720 \pm 190$ 6770 в.c.

$\delta C^{13}=-32.3 \%$

Clay-gyttja from Håkanbol (59 $19^{\circ} \mathrm{N}$ Lat, $14^{\circ} 22^{\prime} \mathrm{E}$ Long), Degerfors, Närke, Sweden. Sample $1840 \mathrm{~cm}$ below surface. Zone V (Jessen). Assumed older than Svea R. Comment: diluted.

\section{U-2091. Håkanbol 5, INS}

$9240 \pm 550$

7290 B.C.

$\delta C^{13}=-33.5 \%$

Clay-gyttja with carbon fragments from Håkanbol $\left(59^{\circ} 10^{\prime} \mathrm{N}\right.$ Lat, $14^{\circ} 22^{\prime}$ E Long), Degerfors, Närke, Sweden, at depth $1830 \mathrm{~cm}$ in same kettle hole filled by peat as U-2080. Zone V (Jessen). At short-lived birch maximum. Assumed older than Svea R. Comment: sample contained more carbon fragments than U-2080. Diluted.

\section{U-2092. Håkanbol 5, H}

$9450 \pm 320$

7500 в.C.

$\delta C^{13}=-31.8 \%$

Humus products extracted from sample used for U-2091. Comment: diluted. 


\section{U-2101. Håkanbol 21, INS}

$9450 \pm 210$

7500 B.c.

$\delta C^{13}=-33.2^{2} / c$

Clay-gyttja from Håkanbol (59 $19^{\prime} \mathrm{N}$ Lat, $14^{\circ} 22^{\prime} \mathrm{E}$ Long), Degerfors, Närke, Sweden, at depth $1825 \mathrm{~cm}$ below surface. Zone V (Jessen). Below start of Alnus. Assumed to be beginning of Svea R. Comment: diluted.

\section{U-2094. Solbergatjärn 110 A, INS}

$8600 \pm 600$

6650 в.C.

Fine-detritus-oyttja from Solbergatjörn $\left(59^{\circ} 19^{\prime} \mathrm{N}\right.$ Lon). Degat, $14^{\circ} 27^{\prime} \mathrm{E}$ Long), Degerfors, Närke, Sweden, at depth $733 \mathrm{~cm}$ below surface. Zone boundary V/VI (Jessen). At beginning of Alnus, which supposedly occurred 8500 B.P. according to investigations by Fromm (1938) and Lundqvist $(1957)$. Comment: diluted.

\section{U.2102. Solbergatjärn $110 \mathrm{~A}, \mathrm{H}$}

$9270 \pm 550$

7320 в.с.

$\delta C^{13}=-33.0 \%$ diluted.

Humus products extracted from sample used for U-2094. Comment:

\section{U.2134. Klippetorpstjärn 125 A, INS}

$9330 \pm 600$ 7380 в.C.

$\delta C^{13}=-30.8 \%$

Fine-detritus-gyttja from Klippetorpstjärn $\left(59^{\circ} 12^{\prime} \mathrm{N}\right.$ Lat, $14^{\circ} 26^{\prime}$ E Long), Degerfors, Närke, Sweden, at depth $638 \mathrm{~cm}$ below surface. Zone boundary V/VI (Jessen). At beginning of Alnus (cf. U-2094). Comment: diluted.

\section{U-2093. Håkanbol 25, INS}

$$
\begin{gathered}
8920 \pm 160 \\
6970 \text { B.c. } \\
\delta C^{13}=-34.8 \%
\end{gathered}
$$

Fine-detritus-gyttja from Håkanbol $\left(59^{\circ} 10^{\prime} \mathrm{N}\right.$ Lat, $14^{\circ} 22^{\prime} \mathrm{E}$ Long), Degerfors, Närke, Sweden, at depth $1800 \mathrm{~cm}$ below surface. Zone boundary V/VI (Jessen). At beginning of Alnus (cf. U-2094 and U-2090, 10 $\mathrm{km}$ away). Comment: diluted.

\section{U-2108. Håkanbol 25, H}

$$
\begin{gathered}
\mathbf{8 6 9 0} \pm \mathbf{5 3 0} \\
\mathbf{6 7 4 0} \text { в.C. } \\
\delta C^{13}=-33.3 \%
\end{gathered}
$$
diluted.

Humus products extracted from sample used for U-2093. Comment:

\section{Land uplift series, Central Sweden}

Sediments from Central Sweden, coll. from ancient lakes developed by isolation from sea (S. Florin, 1944, 1948, 1963). Subm. by S. Florin, Inst. Quaternary Geol., Univ. Uppsala, Sweden. Pollen analyses by Th. Candolin. Several samples were dated previously (Radiocarbon: 1959, v. 1, p. $94 ; 1964$, v. 6 , p. $301-302 ; 1965$, v. 7, p. $322-324 ; 1967$, v. 9 , p. $463-464)$. 


\section{U-648. Nedre Mogetorp, 670813, INS}

$6640 \pm 80$

4690 в.c.

$\delta C^{13}$ assumed $-34.1 \%$

Gyttja with Carex from Nedre Mogetorp $\left(59^{\circ} 00^{\prime} \mathrm{N}\right.$ Lat, $16^{\circ} 09^{\prime} \mathrm{E}$ Long), Södermanland, Sweden, alt $44.8 \mathrm{~m}$, at depth 396 to $400 \mathrm{~cm}$ below surface, Littorina time, before LII maximum. Layer corresponds to 400 $\mathrm{cm}$ depth below surface in diagram given by Florin (1948). Coll. 1967 by S. Florin and Ingrid U. Olsson.

\section{U-2055. Nedre Mogetorp, 670813, H}

$$
\begin{gathered}
\mathbf{5 9 9 0} \pm \mathbf{1 4 0} \\
\mathbf{4 0 4 0} \text { B.c. } \\
\delta C^{13}=-27.3 \%
\end{gathered}
$$

Humus products extracted from sample used for U-648 and U-2115. Comment: diluted.

U.2115. Nedre Mogetorp, 670813, $\mathrm{KMnO}_{4} \quad>17,600$ $\delta C^{13}$ assumed $-25.0 \%$

Gas obtained by wet combustion of sample with $\mathrm{KMnO}_{4}$. Comment: high age indicates that sediment contains old carbon, probably graphite, and that age of U-648 in radiocarbon yr is too high. Diluted.

U-700. Överåda, INS

$2750 \pm 70$

800 B.C.

$\delta C^{13}=-26.5 \%$

Brackish-water gyttja from Överåda $\left(58^{\circ} 53^{\prime} \mathrm{N}\right.$ Lat, $17^{\circ} 30^{\prime} \mathrm{E}$ Long), Vagnhärad-Trosa, Sweden, alt $27.0 \mathrm{~m}$. Sample, $10 \mathrm{~cm}$ long, $150 \mathrm{~cm}$ below surface. Sub-Boreal, Zone VIII (Jessen), assoc. with neolithic pitted ware.

\section{U-702. Överåda, H}

$2080 \pm 60$

130 B.C.

Humus products from sample used for U-700.

\section{Sjödyn series}

Gyttja from tarn Sjödyn (59 $55^{\prime}$ N Lat, $17^{\circ} 42^{\prime}$ E Long), Vaksala parish, Uppland, Sweden, alt $34.7 \mathrm{~m}$. Drainage threshold at $36.8 \mathrm{~m}$. Sediments described by Sidenvall (1967). Depth to post-glacial clay ca. $6.5 \mathrm{~m}$ below water surface, water depth ca. $1.26 \mathrm{~m}$. Livingstone borer used as sampler. Pollen and diatom analyses by Sidenvall. Coll. 1963 and subm. by Jan Sidenvall, Inst. Quaternary Geol. Univ. Uppsala, Sweden. Comment: samples diluted.

\section{U-2047. Sjödyn, 441 to 434 , INS}

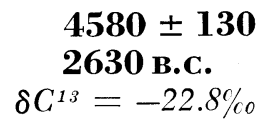

Clay-gyttja, gray-greenish with olive-greenish bands, 441 to $434 \mathrm{~cm}$ below surface at beginning of lagoon stage. Zone VIII (Jessen).

\section{U-2046. Sjödyn 430 to 425 , INS}

$4850 \pm 140$ 2900 B.C. $\delta C^{13}=-26.5 \%$

Gyttja, 430 to $425 \mathrm{~cm}$ below surface from boundary between lagoon 
gyttja and freshwater gyttja, coll. $10 \mathrm{~cm}$ higher than U-2047. Zone VIII (Jessen).

\section{U-2074. Sjödyn 230, INS}

Gyttja with some dy, ca. $230 \mathrm{~cm}$ below surface. Zone boundary VII/ VIII (Jessen). Alnus decreasing and Betula increasing. Carpinus betulus and Fagus silvatica show continuous curves. Sample shows beginning of Picea and of agriculture.

\section{U-2075. Sjödyn 230, H}

Humus products extracted from sample used for U-2074.

$$
\delta C^{13}=-31.8 \%
$$

\section{Floran series}

Gyttja from mire district "Floran" in N Uppland, Sweden, to determine land uplift and to study vegetational history. District described by Ingmar (1963). Previous samples in series are given in Radiocarbon, 1965 , v. 7 , p. 324-326. Pollen analyses by Thorolf Candolin, diatom analyses by Tord Ingmar. Coll. and subm. by Ingmar, Inst. Plant Ecol. Univ. Uppsala, Sweden.

\section{U.2089. Sörbackenmossen 3, INS}

$4650 \pm 130$ 2700 B.c.

Gyttja from bog Sörbackenmossen (60 $20^{\circ} \mathrm{N}$ Lat, $17^{\circ} 42^{\prime}$ E Long), Uppland, Sweden, alt $42 \mathrm{~m}$. Sample, 3-cm-thick layer of brackish-water gyttja, immediately below boundary to freshwater gyttja. Sample grayish brown, somewhat minerogenous. Zone VIII (Jessen). Coll. 1966.

\section{U-2073. Sörbackenmossen 3, H}

$4590 \pm 80$ 2640 B.c.

Humus prdoucts extracted from sample used for U-2089.

$$
\delta C^{13}=-20.1 \%
$$

U-663. Sörbackenmossen 2, INS

$4660 \pm 90$ 2710 B.c.

$$
\delta C^{13}=-22.4 \%
$$

Gyttja from bog Sörbackenmossen $\left(60^{\circ} 20^{\prime} \mathrm{N}\right.$ Lat, $17^{\circ} 42^{\prime} \mathrm{E}$ Long), Uppland, Sweden, alt $42 \mathrm{~m}$. Sample, 3-cm-thick layer of freshwater gyttja, immediately above boundary to brackish-water gyttja. Sample grayish brown, hardly minerogenous. Zone VIII (Jessen). Coll. 1966.

\section{U-2109. Skrivarmyren 2, INS}

$4470 \pm 110$

2520 B.C.

$\delta C^{13}=-16.5 \%$

Gyttja from bog Skrivarmyren $\left(60^{\circ} 20^{\prime} \mathrm{N}\right.$ Lat, $17^{\circ} 44^{\prime} \mathrm{E}$ Long), Uppland, Sweden, drainage threshold at alt $39 \mathrm{~m}$. Sample, 2-cm-thick layer of brackish-water gyttja, just below boundary to freshwater gyttja. Sample dark olive green, somewhat minerogenous and gelatinous. Zone VIII (Jessen). Coll. 1967. 


\section{U-664. Skrivarmyren 1, INS}

Gyttja from bog Skrivarmyren $\left(60^{\circ} 20^{\prime} \mathrm{N}\right.$ Lat, $17^{\circ} 44^{\prime} \mathrm{E}$ Long), Uppland, Sweden, drainage threshold at alt $39 \mathrm{~m}$. Sample, 2-cm-thick layer of freshwater gyttja, immediately above boundary to brachishwater gyttja. Sample reddish-brown, gelatinous Cyanophyceae-gyttja. Zone VIII (Jessen). Coll. 1967.

\section{U-639. Skrivarmyren $1, \mathbf{H}$}

$$
\begin{array}{r}
\mathbf{4 3 5 0} \pm \mathbf{8 0} \\
\mathbf{2 4 0 0} \text { B.c. } \\
\delta C^{13} \text { assumed }-21.4 \% \%
\end{array}
$$

Humus products extracted from sample used for U-664.

\section{U-2025. Ulvsbo trusk 4, INS}

$3690 \pm 100$

1740 B.C.

Gyttja from tarn Ulvsbo trusk $\left(60^{\circ} 19^{\prime} \mathrm{N}\right.$ Lat, $17^{\circ} 46^{\prime} \mathrm{E}$ Long), Uppland, Sweden, drainage threshold at alt $35 \mathrm{~m}$. Sample, 4-cm-thick layer of light-brown and slightly minerogenous gyttja around transition from brackish-water gyttja to freshwater gyttja. Zone VIII (Jessen). Coll. 1965 .

\section{U-704. Ulvsho trusk 3, H}

Humus products extracted from gyttja from tarn Ulvsbo trusk $\left(60^{\circ}\right.$ $19^{\prime} \mathrm{N}$ Lat, $17^{\circ} 46^{\prime} \mathrm{E}$ Long), Uppland, Sweden, drainage threshold at alt $35 \mathrm{~m}$. Sample, 2-cm-thick layer of brackish-water gyttja, immediately below boundary to freshwater gyttja. Sample light-brown and slightly minerogenous. Zone VIII (Jessen). Coll. 1964.

\section{U-2024. Ensjön 2, INS}

$$
\begin{aligned}
& 2950 \pm 170 \\
& 1000 \text { B.C. } \\
& \delta C^{13}=-21.1 \% o
\end{aligned}
$$

Gyttja from lake Ensjön ( $60^{\circ} 24^{\prime} \mathrm{N}$ Lat, $17^{\circ} 51^{\prime} \mathrm{E}$ Long), Uppland, Sweden, drainage threshold at alt $27 \mathrm{~m}$. Sample, 3-cm-thick layer of brackish-water gyttja, immediately below boundary to freshwater gyttja. Sample dark olive green and somewhat minerogenous and gelatinous. Zone VIII (Jessen). Coll. 1964.

\section{U-642. Ensjön 2, H}

$3660 \pm 80$

1710 B.C.

Humus products extracted from sample used for U-2024.

\section{U-537. Västerängen 2, INS 1}

$$
2350 \pm 220
$$

400 B.c.

$\delta C^{13}=-28.2^{\%} \%$

Gyttja from ditched peatland Västerängen $\left(60^{\circ} 25^{\prime} \mathrm{N}\right.$ Lat, $14^{\circ} 44^{\prime}$ E Long), Uppland, Sweden, drainage threshold at alt $20 \mathrm{~m}$. Sample, 1cm-thick layer, uppermost part of brackish-water gyttja rich in Phrag- 
mites, underlying gyttja-free Phragmites peat. Sample represents isolation from sea in this very shallow basin. Sample underlay marked increase of Picea pollen in beginning of Zone IX (Jessen). Coll. 1963.

\section{U-574. Västerängen 2, INS 2}

$2610 \pm 70$

$\delta C^{13}=-26.9 \%$

Gyttja from same sample as U-537, but new pretreatment.

$$
1500 \pm 180
$$

\section{U-2078. Kätjaure}

A.D. 450

$\delta C^{13}=-23.4 \%$

Peat from Kätjaure $\left(67^{\circ} 18^{\prime} \mathrm{N}\right.$ Lat, $17^{\circ} 00^{\prime} \mathrm{E}$ Long), Padjelanta, Lappland, Sweden. Sample, $80 \mathrm{~cm}$ below surface, from frozen peat. Coll. 1967 and subm. by Anders Rapp, Dept. Physical Geog. Univ. Uppsala, Sweden. Comment: diluted.

\section{Latnjajaure series}

Diatom gyttja from Latnjajaure $\left(68^{\circ} 21^{\prime} \mathrm{N}\right.$ Lat, $18^{\circ} 37^{\prime} \mathrm{E}$ Long), Abisko, Lappland, Sweden. Core might contain material washed down from surroundings. Coll. 1967; subm. by Arnold Nauwerck, Inst. Limnology, Univ. Uppsala, Sweden. Comment: inconsistency in results might be due to contamination during storage. All samples diluted. Very little humus was recovered.

\section{U-2086. Latnjajaure, 138.5 to 125 , INS}

$6510 \pm 230$

4560 в.C.

Gyttja from 138.5 to $125 \mathrm{~cm}$ depth in core.

\section{U-2087. Latnjajaure, 138.5 to $125, \mathrm{H}$}

\section{A.D. 1850}

$$
100 \pm 300
$$

$\delta C^{13}$ assumed $-25.2 \%$

Humus products extracted from sample used for U-2086.

\section{U-2084. Latnjajaure, 65 to 55 , INS}

Gyttja from 65 to $55 \mathrm{~cm}$ depth in core.

$$
\begin{gathered}
10,750 \pm \mathbf{3 4 0} \\
\mathbf{8 8 0 0} \text { в.C. } \\
\delta C^{13}=-28.2 \%
\end{gathered}
$$

\section{U-2085. Latnjajaure, 65 to $55, \mathrm{H}$}

5600 в.C.

Humus products extracted from sample used for U-2084.

$$
\delta C^{13} \text { assumed }-25.2 \%
$$

\section{ARCHAEOLOGIC SAMPLES \\ A. Central Europe}

\section{C.611. Budva ship}

Wood from wrecked ship at Budva $\left(42^{\circ} 1 \overline{7}^{\prime} \mathrm{N}\right.$ Lat, $18^{\circ} 50^{\prime}$ E Long), Montenegro, Yugoslavia. Coll. 1966 by V. Stanišić and subm. by A. 
Sliepčević, Institute Ruder Bošković, Zagreb, Yugoslavia. Comment: another portion of same sample was dated as a quick check and also gave very low age.

\section{Gårdlösa series}

B. Sweden

Charcoal from Gårcllösa grave-field No. 2 and No. $3\left(55^{\circ} 34^{\prime} \mathrm{N}\right.$ Lat, $14^{\circ} 08^{\prime}$ E Long), Smedstorp parish, Skåne, Sweden. Coll. and subm. by Berta Stjernquist, Inst. Scandinavian Antiquities, Univ. Lund, Sweden. Several samples were dated previously (Radiocarbon: 1965, v. 7, p. 326327 ; 1967 , v. 9, p. 465-467).

Charcoal found in bottom of hearth, 0.15 to $9.20 \mathrm{~m}$ below big stone in setting. Coll. 1967.

U-672. Gårdlösa 2, Stone-settings 1967, No. $67: 32$

$$
\begin{aligned}
& \mathbf{1 3 4 0} \pm \mathbf{7 0} \\
& \text { A.D. } 610 \\
& \delta C^{13}=-26.5 \% \circ
\end{aligned}
$$

Charcoal from bottom at 0.58 to $0.63 \mathrm{~m}$ depth with bones of a dog. Coll. 1967.

U-670. Gårdlösa 3, House XLIX

$$
\begin{gathered}
1170 \pm \mathbf{6 0} \\
\text { A.D. } 780 \\
\delta C^{13}=-22.8 \%
\end{gathered}
$$

Charcoal, at depth 0.75 to $0.85 \mathrm{~m}$, below surface with potsherds, metal objects, etc. Coll. 1966. Comment: upper $0.2 \mathrm{~m}$ of profile cultivated.

\section{U-671. Gårdlösa 3, Grave 36}

$2570 \pm 80$

620 B.C.

$$
\delta C^{13}=-25.4 \%
$$

Charcoal from 0.4 to $0.8 \mathrm{~m}$ depth, from post-hole below cairn, where Grave 36 was located. Coll. 1966.

\section{U-674. Gårdlösa 3, Grave 42}

$$
\begin{gathered}
\mathbf{1 3 0 0} \pm \mathbf{8 0} \\
\text { A.D. } 650 \\
\delta C^{13}=-26.8 \% 0
\end{gathered}
$$

Charcoal from 0.35 to $0.55 \mathrm{~m}$ depth, from layer with charcoal and soot; found with metal objects. Coll. 1965. Comment: upper $0.15 \mathrm{~m}$ cultivated.

\section{U-673. Sandby 11, Well}

$$
\begin{aligned}
& \mathbf{1 3 9 0} \pm \mathbf{7 0} \\
& \text { A.D. 560 } \\
& \delta C^{13}=-24.0 \%
\end{aligned}
$$

Wood from Sandby (54 $26^{\prime} \mathrm{N}$ Lat, $14^{\circ} 12^{\prime}$ E Long), Borrby parish, Skåne, Sweden. Sample from oak-log, $0.73 \mathrm{~m}$ deep covered by $0.30 \mathrm{~m}$ stones. A hearth was on log. Coll. 1965. Comment: upper $0.2 \mathrm{~m}$ cultivated.

\section{Stora Alvaret series}

Charcoal from Mysinge 1:9, 1:3 (56 $32^{\prime} \mathrm{N}$ Lat, $16^{\circ} 3012^{\prime}$ E Long), öland, Sweden. Iron fibula indicate usage of settlement to ca. A.D. 300 . 
Charcoal pieces sorted to allow determination of different bush and tree species. Coll. 1966 and subm. by Ebba-Stina Königsson, Inst. Nordic Antiquities, Univ. Uppsala, Sweden.

\section{U-654. Mysinge, F 114:1}

$1430 \pm 70$

Charcoal, of undetermined specie, $0.5 \mathrm{~m}$ below surface, overlying bedrock close to wall of one "house," A:4.

\section{U-2063. Mysinge, F 114:2}

$$
\begin{aligned}
& \quad 1630 \pm 100 \\
& \text { A.D. 320 } \\
& \delta C^{13}=-19.0 \%
\end{aligned}
$$

Rhamnus cathartica from same place and level as U-654.

\section{U-653. Mysinge, F 124:1}

$$
1710 \pm 70
$$

A.D. 240

$\delta C^{13}=-24.0 \%$

Charcoal, of undetermined specie, $0.5 \mathrm{~m}$ below surface of "house" A:2, close to sample U-654.

\section{U.649. Mysinge, F 124:2}

$$
1800 \pm 80
$$

$$
\text { A.D. } 150
$$

$\delta C^{13}=-25.6 \%$

Betula and Populus from same place and level as U-653.

\section{U-662. Mysinge F 124:3}

$$
1640 \pm 70
$$

Juniperus and Taxus from same place and level as U-653.

\section{Västerås-Äs series}

Charcoal from $\ddot{A s}\left(59^{\circ} 44^{\prime} \mathrm{N}\right.$ Lat, $16^{\circ} 30^{\prime}$ E Long), Romfartuna parish, Västmanland, Sweden, undisturbed cultural layer containing pitted pottery. Coll. 1967 and subm. by Lars Löfstrand, Inst. Nordic Antiquities, Univ. Uppsala, Sweden.

\section{U-2112. Västerås-Äs 785/396}

$$
\begin{gathered}
1120 \pm 70 \\
830 \text { B.C. } \\
\delta C^{13}=-25.5 \%
\end{gathered}
$$

Charcoal from lowest part of Layer G-S II, at ca. 15 to $20 \mathrm{~cm}$ depth, dating Stå MN pitted pottery culture.

\section{U-701. Västerås-ёs 841/394}

$$
2250 \pm 60
$$

300 B.c.

Charcoal from hearth in middle of Layer G-S II, at ca. $15 \mathrm{~cm}$ depth, dating ornamented pitted pottery of Säter III type.

\section{Berthåga series}

Charcoal from dwelling under Iron age grave-field. Site excavated in connection with new grave-field, Berthåga kyrkogård $\left(59^{\circ} 51 \mathrm{~N}\right.$ Lat, 
$17^{\circ} 35^{\prime}$ E Long), Uppland, Sweden. Coll. and subm. by Astrid Sjöberg, Inst. Nordic Antiquities, Univ. Uppsala, Sweden.

\section{U-656. Berthåga A212, F2}

Charcoal from burned piece of wood, ca. $40 \times 10 \mathrm{~cm}$, from Hearth Pit A212, also containing animal tooth and burned clay. Coll. 1967.

\section{U-657. Berthåga A218, F2}

$$
\begin{gathered}
2390 \pm 80 \\
440 \text { в.C. }
\end{gathered}
$$

Charcoal from concentration in layer with charcoal and soot at bottom of split-stone mound containing potsherd and burned clay, at ca. $0.5 \mathrm{~m}$ depth. Coll. 1967.

\section{U-658. Berthåga A216, F71}

\section{A.D. 1850}

$100 \pm 70$

$\delta C^{13}=-24.8 \%$

Charcoal from layer with charcoal and soot, at 0.30 to $0.35 \mathrm{~m}$ depth. Nearby were house-remains, post-holes, burned clay, animal bones, and potsherds. Coll. 1966.

\section{U-684. Berthåga A216, R214296}

A.D. 1870

$80 \pm 110$

Charcoal from same layer as U-658. Coll. 1966.

\section{U-688. Avasjön}

$$
\begin{aligned}
& \quad \mathbf{4 6 0} \pm \mathbf{5 0} \\
& \text { A.D. } 1490 \\
& \delta C^{13}=-24.3 \%
\end{aligned}
$$

Wood from SE end of Avasjön (64. $14^{\prime} \mathrm{N}$ Lat, $21^{\circ} 20^{\prime} \mathrm{E}$ Long), Lövånger parish, Västerbotten, Sweden, from board of ship, ca. $10 \mathrm{~m}$ long. Part of ship is still in lake. Avasjön supposedly is part of old passage; 4 wrecks are known. Commercial center at Mångbyn was flourishing ca. 450 yr ago. Coll. 1926: subm. by Peter Gustavsson, Inst. Nordic Antiquities, Univ. Uppsala, Sweden.

\section{Yttervik series}

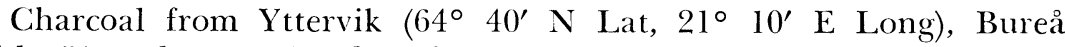
parish, Västerbotten, Sweden, from burial cairn. Described by Westin (1962). Coll. 1875 by Hans Hildebrand, Central Office Natl. Antiquities, Stockholm, Sweden; subm. by Hans Christiansson, Inst. Nordic Antiquities, Univ. Uppsala, Sweden. Samples stored, since coll. in Mus. Antiquities, Stockholm, Sweden.

\section{U-2057. Yttervik 5}

$$
640 \pm 90
$$

\section{A.D. 1310}

$\delta C^{13}=-19.0 \%$

Charcoal from Grave 5, a stone-setting. No other artifacts found. Grave $10 \mathrm{~m} \mathrm{~W}$ of old road to Båtvik. Collection No. SHM 7043 B. 


\section{U-655. Yttervik 9}

Charcoal from Grave 9, a cairn. No other artifacts found. Grave ca. $5 \mathrm{~m} \mathrm{E}$ of old road to Båtvik. Collection No. SHM $7043 \mathrm{~B}$.

\section{U.2070. Yttervik 12}

$$
\begin{aligned}
& \quad 1110 \pm 100 \\
& \text { A.D. 840 } \\
& \delta C^{13}=-35.7 \% 0
\end{aligned}
$$

Charcoal from Grave 12, a cairn. No other artifacts found. Grave ca. $10 \mathrm{~m} \mathrm{E}$ of old road to Båtvik. Collection No. SHM 7043 B. Comment: diluted.

\section{Bjurselet series}

Charcoal from Bjurselet $\left(65^{\circ} 00^{\prime} \mathrm{N}\right.$ Lat, $21^{\circ} 04^{\prime} \mathrm{E}$ Long), Byske parish, Västerbotten, Sweden. From Stone age dwelling-site, alt $53 \mathrm{~m}$, with imported flint axes, scrapers, and potsherds. Subm. by Hans Christiansson. Upper Layer consisted of cultivated soil, Layer I, underlain by sand, Layers II, III, and IV. Preliminary described by Christiansson $(1965 \mathrm{a}, \mathrm{b})$. Pollen profile from same area and several archaeologic samples were dated previously (Radiocarbon, 1967, v. 9, p. 467-469).

\section{U-647. Bjurselet 210/228 Åkern}

$$
\begin{gathered}
2140 \pm 80 \\
190 \text { B.c. } \\
\delta C^{13}=-24.7 \% 0
\end{gathered}
$$

Charcoal, from field, from lowest layer with brittle burned stones. Coll. 1966 by P. Gustavsson, Inst. Nordic Antiquities, Univ. Uppsala, Sweden.

\section{U-2035. Bjurselet, Hunting Pit 1, Svarvet}

$$
\begin{array}{r}
930 \pm 190 \\
\text { A.D. } 1020 \\
\delta C^{13}=-25.8 \% \circ
\end{array}
$$

Charcoal, at $0.9 \mathrm{~m}$ depth, from hunting pit, $1.2 \mathrm{~m}$ deep. Coll. 1965 by Christiansson. Comment: similar pits recorded in Radiocarbon, 1967, v. 9, p. 469. Diluted.

\section{U-2045. Bjurselet 216/119 Svarvet}

$4310 \pm 180$ 2360 B.C.

$$
\delta C^{13}=-25.1 \%
$$

Wood and charred wood from Layer II in sandy soil, probably never cultivated. Coll. 1965 by H. C. Vorting. Comment: diluted.

$$
\text { III. TREE-RING SAMPLES }
$$

\section{Northern Sweden series}

Wood from N Sweden. Tree felled in 1966, had 75 tree rings. Sample from ca. $1 \mathrm{~m}$ above ground. Subm. 1966 by Ing. A. Assarsson, Mo and Domsjö AB, Örnsköldsvik, Sweden, where chemical treatment was made. Wood was pulverized, water was removed by acetone, and pulverized wood was treated by ethanol-benzene (1:2) mixture for $20 \mathrm{hrs}$ and washed with water for $20 \mathrm{hrs}$. Extract from acetone and ethanol-benzene treat- 
ment was evaporated at temp. $<40^{\circ} \mathrm{C}$, dissolved in di-ethyl-ether, and dried. Part of remaining material was treated with Na-chlorite and acetic acid at elevated temp. to give cellulose and holocellulose (Wise et al., 1946). Results will be discussed by Assarsson and Olsson.

General Comment: extract has excess of $\mathrm{C}^{14}$ relative to air for rather young tree rings but is depleted for oldest rings. Sample U-598 indicates that this wood is, to some extent, affected by younger material. Difference between the 3 fractions of youngest sample is not significant since relative amount of material produced during 1960-1966 probably varied from year to year and "atomic bomb effect" changed greatly.

Table 1

\begin{tabular}{|c|c|c|c|c|c|}
\hline \multirow[b]{2}{*}{ No. } & \multirow[b]{2}{*}{ Sample } & \multirow[t]{2}{*}{$\delta \mathrm{C}^{13} / / 0$} & \multicolumn{3}{|c|}{$\Delta$ in $\% ; ; 1 / 2=5730 \mathrm{yr}$} \\
\hline & & & Extract & $\begin{array}{l}\text { Extracted } \\
\text { wood }\end{array}$ & $\begin{array}{l}\text { Holo- } \\
\text { cellulose }\end{array}$ \\
\hline$\overline{\mathrm{U}}-643$ & $\begin{array}{l}\text { Tree-ring } 69-75, \\
\text { xylem }\end{array}$ & -24.5 & & & $620 \pm 9$ \\
\hline $\begin{array}{l}\text { U-644 } \\
\text { U-645 }\end{array}$ & formed 1960-1966. & $\begin{array}{l}-32.0 \\
-27.9\end{array}$ & $554 \pm 8$ & $645 \pm 10$ & \\
\hline U-626 & $\begin{array}{l}\text { Tree-ring 59-69, } \\
\text { xylem }\end{array}$ & -21.9 & & & $-27 \pm 7$ \\
\hline $\begin{array}{l}\text { U-627 } \\
\text { U-628 }\end{array}$ & formed 1950-1955. & $\begin{array}{l}-25.1 \\
-28.5\end{array}$ & $119 \pm 7$ & $-31 \pm 6$ & \\
\hline $\mathrm{U}-594$ & $\begin{array}{l}\text { Tree-ring } 44-49, \\
\text { xylem }\end{array}$ & -20.7 & & & $-28 \pm 7$ \\
\hline $\begin{array}{l}\text { U-595 } \\
\text { U-596 }\end{array}$ & formed 1935-1941. & $\begin{array}{l}-25.0 \\
-28.2\end{array}$ & $117 \pm 6$ & $-21 \pm 5$ & \\
\hline $\mathrm{U}-597$ & $\begin{array}{l}\text { Tree-ring 26-31, } \\
\text { xylem }\end{array}$ & -23.7 & & & $-22 \pm 6$ \\
\hline U-598 & $\begin{array}{l}\text { formed 1917-1922, } \\
\text { heartwood }\end{array}$ & -25.2 & & $7 \pm 5$ & \\
\hline U-599 & formed 1960-1965. & -26.8 & $10 \pm 5$ & & \\
\hline U-629 & $\begin{array}{l}\text { Tree-ring } 0-10 \text {, } \\
\text { xylem }\end{array}$ & -23.2 & & & $8 \pm 6$ \\
\hline U-630 & $\begin{array}{l}\text { formed 1891-1901, } \\
\text { heartwood. }\end{array}$ & -25.7 & & $13 \pm 5$ & \\
\hline U-63 I & & -26.2 & $-14 \pm 6$ & & \\
\hline
\end{tabular}

\section{Argentina series}

Tree trunk (Fitzroya cupressoides) from Parque Nac. Los Alerces (43 $\mathrm{S}$ Lat, $71^{\circ} \mathrm{W}$ Long), Esquel, Chubut, Argentina. Park created 1937. Tree probably felled a few yr earlier. Trunk donated by Intendencia, Park Nac. Los Alerces through Åke Vinterbäck, Univ. Uppsala, Sweden. 
Comment: tree-ring project is performed in collaboration with Hendrik de Waard, J. C. Vogel, and J. C. Lerman, Natuurkundig Lab. Groningen, The Netherlands. Tree rings counted by Horring, High School of Forestry, Stockholm, Sweden. In the calculations it was assumed that tree was felled A.D. 1934. Samples will be checked for $\mathrm{C}^{13} / \mathrm{C}^{12}$ ratio in Groningen. Cellulose and lignin prepared acc. to Olson and Broecker (1958) after normal pretreatment of wood.

\begin{tabular}{|c|c|c|c|c|c|c|}
\hline Lab. no. & $\begin{array}{l}\text { Age before } \\
\text { A.D. } 1934\end{array}$ & $\begin{array}{c}\text { Age в.P. } \\
(1950)\end{array}$ & Substance & $\delta \mathrm{C}^{13} \% / / 0$ & $\delta \mathrm{C}^{14} \%$ & $\Delta \%$ \\
\hline U-509 & $649-645$ & 663 & I innin & -24.2 & -10.5 & $-12 \pm$ \\
\hline U-650 & $644-640$ & 658 & Lignin & -23.4 & -20.1 & $-23 \pm 5$ \\
\hline U-508 & 634-630 & 648 & Cellulose & -21.3 & -17.3 & $-25 \pm 5$ \\
\hline U-668 & $629-625$ & 643 & Cellulose & -22.9 & -16.5 & $-21 \pm 5$ \\
\hline U-667 & $629-625$ & 643 & Xylem & -24.1 & -13.1 & $-15 \pm 5$ \\
\hline
\end{tabular}

\section{Lake sediment series}

IV. GEOCHEMICAL SAMPLES

Living plants from hard-water lakes coll. to demonstrate influence of dissolved carbonate on $\mathrm{C}^{14} / \mathrm{C}^{12}$ ratio in plants, components of sediments studied on öland. Present $\mathrm{C}^{14} / \mathrm{C}^{12}$ ratio of atmosphere will be given in Radiocarbon, 1970, v. 12. Subm. by Lars-König Königsson, Inst. Quaternary Geol., Univ. Uppsala, Sweden.

\section{U.651. Carex 270666, D}

$$
\begin{array}{r}
\delta \mathbf{C}^{14}=+689 \pm 13 \% \\
\Delta=+\mathbf{7 0 0} \pm \mathbf{1 3} \% \\
\delta C^{13}=-28.1 \%
\end{array}
$$

Carex elata from Dröstorps mose $\left(56^{\circ} 35^{\prime} \mathrm{N}\right.$ Lat, $16^{\circ} 32^{\prime} \mathrm{E}$ Long), öland, Sweden. Coll. June 27, 1966 by Königsson and B. Fredskild. Comment: another portion of this sample was measured by J. M. Punning, TA-222: $\delta \mathrm{C}^{14}=616 \%$.

\section{U-2083. Floating 270666, D}

$$
\begin{array}{r}
\delta \mathbf{C}^{14}=+\mathbf{5 3 3} \pm \mathbf{1 2} \% \\
\Delta=+\mathbf{5 5 6} \pm \mathbf{1 2} \% \\
\delta C^{13}=-32.4 \%
\end{array}
$$

Floating plants from Dröstorps mose ( $56^{\circ} 35^{\prime} \mathrm{N}$ Lat, $16^{\circ} 32^{\prime} \mathrm{E}$ Long), Öland, Sweden. Coll. June 27, 1966 by Königsson and Fredskild.

\section{U-2072. Submerged 270666, D}

$$
\begin{array}{r}
\delta \mathbf{C}^{14}=+561 \pm \mathbf{1 2} \%{ }_{0} \\
\Delta=+\mathbf{5 7 9} \pm \mathbf{1 2} \% \\
\delta C^{13}=-30.9 \%
\end{array}
$$

Submerged plants from Dröstorps mose $\left(56^{\circ} 35^{\prime} \mathrm{N}\right.$ Lat, $16^{\circ} 33^{\prime} \mathrm{E}$ Long), öland, Sweden. Coll. June 27, 1966 by Königsson and Fredskild.

\section{U-652. Characeae and submerged 270666, D}

$$
\begin{array}{r}
\delta \mathbf{C}^{14}=+468 \pm 9 \% \\
\Delta=+457 \pm 9 \% \\
\delta C^{13}=-21.4 \%
\end{array}
$$

Characeae and other submerged plants from Dröstorp mose $\left(56^{\circ}\right.$ 
$35^{\prime} \mathrm{N}$ Lat, $16^{\circ} 32^{\prime} \mathrm{E}$ Long), öland, Sweden. Coll. June 27, 1966 by Königsson and Fredskild.

\section{U-2068. Characeae 270666, BK}

$$
\begin{array}{r}
\delta \mathbf{C}^{14}=+396 \pm 12 \% \\
\Delta=+406 \pm 12 \% \\
\delta C^{13}=-28.7 \%
\end{array}
$$

Characeae from Bårby Källa (Lilla Dammen) $\left(56^{\circ} 31^{\prime} \mathrm{N}\right.$ Lat, $16^{\circ} 30^{\prime}$ E Long), Öland, Sweden. Coll. June 27, 1966 by Königsson and Fredskild.

\section{U-2118. Algae 020768, MM}

$$
\begin{array}{r}
\delta \mathbf{C}^{14}=+\mathbf{3 2 7} \pm \mathbf{1 0} \% \\
\Delta=+\mathbf{3 4 1} \pm \mathbf{1 0} \% \\
\delta C^{13}=-30.2 \%
\end{array}
$$

Algae from Möckelmosen (56 $36^{\prime} \mathrm{N}$ Lat, $16^{\circ} 31^{\prime} \mathrm{E}$ Long), öland, Sweden. Coll. July 2, 1968 by Königsson.

\section{Resin sample series}

Birch-bark from Nåsten (ca. 59 $49^{\prime} \mathrm{N}$ Lat, $17^{\circ} 35^{\prime} \mathrm{E}$ Long), Uppland, Sweden. Tree felled 1967; bark coll. 1968 by Ingrid U. Olsson. Tree ca. $30 \mathrm{yr}$ old. Resin, used for tightening of vessels and fixing of metal objects to wood, etc. is supposed to derive from birch-bark (Sandermann, 1965). Chemical analysis and infra-red spectra of different extracts of archaeologic resin and of resin obtained by heating, at $400^{\circ} \mathrm{C}$, of outer parts of birch-bark, agree so well that origins might be identical. In Swedish resin some part of inner bark might have been used. Comment: results indicate that resin dates would be systematically somewhat too high-the error dependent on age of tree. Only one pair of sample of resin-charcoal has been dated in Uppsala, U-151 and U-152: $2070 \pm 90$ and $2240 \pm 90$, respectively (Deevey et al., 1967, p. 177). All archaeologic resin samples (15), dated in Uppsala, have their $\delta \mathrm{C}^{13}$ between -26 and $-34 \%$.

\section{U.2116. Birch-bark, white, 09068, Ac.}

$$
\begin{array}{r}
\delta \mathbf{C}^{14}=+189 \pm 11 \% \\
\Delta=+191 \pm \mathbf{1 1} \% \\
\delta C^{13}=-25.9 \%
\end{array}
$$

Acetone extract from outer layer of birch-bark.

$$
\begin{array}{r}
\delta \mathbf{C}^{14}=+\mathbf{3 1 9} \pm \mathbf{1 0} \% \\
\Delta=+\mathbf{3 3 0} \pm \mathbf{1 0} \% \\
\delta C^{13}=-29.2 \%
\end{array}
$$

Remains of birch-bark after acetone extraction and normal pretreatment.

\begin{tabular}{ll} 
Date lists: & \multicolumn{1}{c}{ REFERENCES } \\
Copenhagen VII & Tauber, 1966 \\
Uppsala I & Olsson, 1959 \\
Uppsala II & Olsson, 1960 \\
Uppsala III & Olsson et al., 1961 \\
Uppsala IV & Olsson and Kilicci, 1964 \\
Uppsala V & Olsson and Piyanuj, 1965 \\
Uppsala VII & Olsson et al., 1967
\end{tabular}

Balchin, W. G. V., 1941, The raised features of Billefjord and Sassenfjord West Spitsbergen: Geog. Jour., v. 97, p. 364-376. 
Bárdarson, G. B., 1910, Traces of changes of climate and level at Húnaflói, N-Iceland: Postglaziale Klimaveränderungen, Internat. Geol. Kongr., Stockholm, p. 347-352. v. $1,118 \mathrm{p}$.

Birkenmajer, Krzysztof, 1960, Raised marine features of the Hornsund area, Vestspitsbergen: Studia Geol. Polon., v. 5, 95 p.

Christiansson, Hans, 1965a, De arkeologiska undersökningarna vid Bjurselet i Byske: Västerbotten, p. 191-202.

1965b, Flint finds in Västerbotten; Hunting, trade or agriculture?, in: Hunting and Fishing, Nordic symposium in Luleå 1962, Norrbottens Mus., Luleă, Sweden, p. 91-110.

Craig, Harmon, 1961, Mass-spectrometer analyses of radiocarbon standards: Radio carbon, v. 3, p. $1-3$.

Dahl, Ragnar, 1967, Senglaciala ackumulationsformer och glaciationsförhållanden in Narvik-Skjomenområdet, Norge: Norsk geog. tidskr., v. 21, p. 157-241.

1968, Glacial accumulations, drainage and ice recession in the NarvikSkjomen district, Norway: Norsk geog. tidsskr., v. 22, p. 101-165.

Deevey, E. S., Flint, R. F., and Rouse, I. (eds.), 1967, Radiocarbon measurements: Comprehensive index, 1950-1965, Yale Univ., New Haven, $221 \mathrm{p}$.

Einarsson, Thorleifur, 1961, Pollenanalytische Untersuchungen zur spät- und postglazialen Klimageschichte Islands: Sonderöff. Geol. Inst. Univ. Köln, v. 6, 52 p.

Eriksson, K. G. and Olsson, I. U., 1963, Some problems in connection with $\mathrm{C}^{14}$ dating of tests of Foraminifera: Geol. Inst. Univ. Bull., Uppsala, v. 42, p. 1-13.

1967, Några data från en planerad undersökning av en skalbank vid Linddalsskogen, Väjern, Mellersta Bohuslän: Teknik och natur, Studier tillägnade Gunnar Beskow, Akademiförlaget, Göteborg, p. 149-166.

Feyling-Hanssen, R. W., 1955, Stratigraphy of the marine late-Pleistocene of Billefjorden, Vestspitsbergen: Norsk Polarinst. Skr. 107, $186 \mathrm{p}$.

Feyling-Hanssen, R. W. and Olsson, Ingrid, 1959-1960, Five radiocarbon datings of post glacial shorelines in central Spitsbergen: Norsk geog. tidsskr., v. 17, p. 122-131.

Florin, Sten, 1944, Havsstrandens förskjutningar och bebyggelseutvecklingen i östra Mellansverige under senkvartär tid. I. Allmän översikt: G. F. F., v. 66, p. 551-634 1948, Havsstrandens förskjutningar och bebyggelseutvecklingen i östra Mellansverige under senkvartär tid. II. De baltiska strandbildningarna och stenåldersboplatsen vid Dammstugan nära Katrineholm: G. F. F., v. 70, p. 17-202 v. 1, p. 233-264.
1963schwankungen i Schweden während des Spätquartärs: Baltica,

Fredén, Curt, 1967, A historical review of the Ancylus Lake and the Svea River: G. F. F., v. 89, p. 239-267.

Fromm, Erik, 1938, Geochronologisch datierte Pollendiagramme und Diatoméenanalysen aus Ångermanland: G. F. F., v. 60, p. 365-381. Hoppe, Gunnar, 1965, Submarine peat in the Shetland Islands: Geog. Annales, v. 47,
ser. A, p. 195-203.

Hospers, J., 1953, Reversals of the main geomagnetic field I: Akad. Sci. Amsterdam, Proc. ser. B, v. 56, p. 467-476.

Hyvärinen, Hannu, 1968, Late-quaternary sediment cores from lakes on Bjørnøya: Geog. Annales, v. 50, ser. A, p. 235-245.

Ingmar, Tord, 1963, Från havsvik till mosse: Sveriges Natur, Årsbok, v. 54, p. 155-177.

Libby, W. F., 1955, Radiocarbon dating, 2nd ed.: Chicago, Univ. of Chicago Press, 175 p.

Lundqvist, Gösta, 1957, C ${ }^{14}$-analyser i svensk kvartärgeologi 1955-57 (with English summary): Sveriges geol. undersökning, ser. C, no. 557, v. 51, no. 8, 25 p.

Norin, Erik, 1966, Edsengol-oasen i Gobiöknen: G. F. F., v. 88, p. 340-350.

Olson, E. A. and Broecker, W. S., 1958, Sample contamination and reliability of radiocarbon dates: N.Y. Acad. Sci. Trans. ser. II, v. 20, p. 593-604.

Olsson, Ingrid, 1958, A $\mathrm{C}^{14}$ dating station using the $\mathrm{CO}_{2}$ proportional counting method: Arkiv f. Fysik, v. 13, p. 37-60.

1959, Uppsala natural radiocarbon measurements I: Am. Jour. Sci. Radiocarbon Suppl., v. 1, p. 87-102.

1960, Uppsala natural radiocarbon measurements II: Am. Jour. Sci. Radiocarbon Suppl., v. 2, p. 112-128.

Olsson, Ingrid, Cazeneuve, Horacio, Gustavsson, John, and Karlén, Ingvar, 1961, Uppsala natural radiocarbon measurements III: Radiocarbon, v. 3, p. 81-85. 
Olsson, I. U., 1965, Computer calculations of $\mathrm{C}^{14}$ determinations: Proc., 6th internatl. conf. radiocarbon and tritium dating, Pullman, Washington, June 7-11, 1965, p. $383-392$.

1966, Computer calculations of $\mathrm{C}^{14}$ determinations: Uppsala Univ. Inst. Physics Rep., UUIP-477, 11 p.

1968, The radiocarbon analyses of lake sediment samples from Bjørnøya: Geog. Annales, v. 50, ser. A, p. 246-247.

Olsson, I U. and Blake, Weston, Jr., 1961-1962, Problems of radiocarbon dating of raised beaches, based on experience in Spitsbergen: Norsk geog. tidsskr., v. 18, p. $47-64$.

Olsson, I. U. and Eriksson, K. G., 1965, Remarks on $\mathrm{C}^{14}$-dating of shell material in sea sediments: Progr. in Oceanog., v. 3, p. 253-266.

Olsson, I. U., Göksu, Yeter, and Stenberg, Allan, 1968, Further investigations of storing and treatment of foraminifera and mollusks for $\mathrm{C}^{14}$-dating: G. F. F., v. 90, p. 417-426.

Olsson, I. U. and Kilicci, Serap, 1964, Uppsala natural radiocarbon measurements IV: Radiocarbon, v. 6, p. 291-307.

Olsson, I. U. and Piyanuj, Piya, 1965, Uppsala natural radiocarbon measurements V: Radiocarbon, v. 7, p. 315-330.

Olsson, I. U., Stenberg, Allan, and Göksu, Yeter, 1967, Uppsala natural radiocarbon measurements VII: Radiocarbon, v. 9, p. 454-470.

Sandermann, Wilhelm, 1965, Untersuchung vorgeschichtlicher "Gräberharze" und Kitte: Techn. Beitr. Archäol., v. 2, p. 58-73.

Sidenvall, Jan, 1967, Senkvartär vegetationsutveckling i trakten av Sjödyn, Vaksala socken, Uppland: Inst. Ouaternary Geol., Uppsala, 16 p.

Sjöberg, Astrid, 1969, A Bronze Age site at Berthåga: Tor, 1967/1968, v. 12, p. 243-247.

Tauber, Henrik, 1966, Copenhagen radiocarbon dates VIII: Radiocarbon, v. 8, p. 213-234.

Thorarinsson, Sigurdur, 1955, The Nucella shore line at Húnaflói in the light of tephrochronological and radiocarbon datings: Náttúrufraedingurinn, v. 25, p. 172-186.

Todd, Ruth, 1958, Foraminifera from Western Mediterranean deep-sea cores: Rept. Swedish Deep-Sea Exped., v. 8, no. 3, p. 167-217.

Westin, Gunnar, 1962, Övre Norrlands forntid. Övre Norrlands historia I. (Umeå), p. $1-122$.

Wise, L. E., Murphy, Maxine, and D'Addieco, A. A., 1946, Chlorite holocellulose, its fractionation and bearing on summative wood analysis and on studies on the hemicelluloses: Paper Trade Jour., v. 122, p. 35-43. 